PROCEEDINGS OF THE

AMERICAN MATHEMATICAL SOCIETY

Volume 134, Number 10, October 2006, Pages 2885-2893

S 0002-9939(06)08295-5

Article electronically published on April 7, 2006

\title{
A CLASS OF NEVANLINNA FUNCTIONS RELATED TO SINGULAR STURM-LIOUVILLE PROBLEMS
}

\author{
SEPPO HASSI, MANFRED MÖLLER, AND HENK DE SNOO
}

(Communicated by Joseph A. Ball)

\begin{abstract}
The class of Nevanlinna functions consists of functions which are holomorphic off the real axis, which are symmetric with respect to the real axis, and whose imaginary part is nonnegative in the upper halfplane. The Kac subclass of Nevanlinna functions is defined by an integrability condition on the imaginary part. In this note a further subclass of these Kac functions is introduced. It involves an integrability condition on the modulus of the Nevanlinna functions (instead of the imaginary part). The characteristic properties of this class are investigated. The definition of the new class is motivated by the fact that the Titchmarsh-Weyl coefficients of various classes of Sturm-Liouville problems (under mild conditions on the coefficients) actually belong to this class.
\end{abstract}

\section{INTRODUCTION}

The class $\mathbf{N}$ of Nevanlinna functions is the collection of functions $Q(z)$ which are holomorphic on $\mathbb{C} \backslash \mathbb{R}$, have the symmetry property $\overline{Q(z)}=Q(\bar{z})$, and for which the kernel

$$
\frac{Q(z)-\overline{Q(w)}}{z-\bar{w}}, \quad z, w \in \mathbb{C} \backslash \mathbb{R}, \quad z \neq \bar{w}
$$

is nonnegative. The general Nevanlinna functions $Q(z) \in \mathbf{N}$ coincide with the Titchmarsh-Weyl coefficients of $2 \times 2$ trace-normed canonical systems of differential equations on the half-line $[0, \infty)$; see [1]-[4], and also [13]. The Kac class $\mathbf{N}_{1}$ is the collection of all Nevanlinna functions $Q(z)$ for which

$$
\int_{1}^{\infty} \frac{\operatorname{Im} Q(i y)}{y} d y<\infty
$$

The subclass $\mathbf{N}_{0}$ of $\mathbf{N}_{1}$ consists of all Nevanlinna functions $Q(z)$ for which

$$
\sup _{y>0} y \operatorname{Im} Q(i y)<\infty .
$$

For more on these classes, see [1], 12, [7, Section 1]. It has been shown recently (see [8, Theorem 3.1], 9, Theorem 4.1]) that under mild conditions all but one

Received by the editors August 3, 2004 and, in revised form, April 12, 2005.

2000 Mathematics Subject Classification. Primary 30D15; Secondary 34B20.

Key words and phrases. Nevanlinna function, Kac class, integral representation, SturmLiouville problem, Titchmarsh-Weyl coefficient.

The authors gratefully acknowledge the support of the NRF of South Africa under GUN 2053746, of the Research Institute for Technology of the University of Vaasa, and of the Dutch Association for Mathematical Physics under MF04/62a. 
of the Titchmarsh-Weyl coefficients of a Sturm-Liouville operator $-D p D+q$ on the half-line $[0, \infty)$ in the limit-point case belong to the Kac class $\mathbf{N}_{1}$. This statement remains valid for a class of Sturm-Liouville problems where the eigenvalue parameter appears rationally in the leading coefficient; cf. [10, Theorem 5.1].

In fact, the arguments in [8, 9], 10] show that the corresponding TitchmarshWeyl coefficients have additional properties. To describe these properties, the class $\mathbf{M}_{1}$ is introduced, which consists of all functions $Q(z) \in \mathbf{N}$ for which there exists a number $c \in \mathbb{R}$ such that

$$
\int_{1}^{\infty} \frac{|Q(i y)-c|}{y} d y<\infty
$$

This class parallels the class $\mathbf{M}_{0}$ of all $Q(z) \in \mathbf{N}$ for which there exists a number $c \in \mathbb{R}$ such that

$$
\sup _{y>1} y|Q(i y)-c|<\infty .
$$

In this note the characteristic properties of functions in the classes $\mathbf{M}_{1}$ and $\mathbf{M}_{0}$ are investigated.

\section{NEVANLINNA FUNCTIONS}

Recall that $Q(z) \in \mathbf{N}$ if and only if $Q(z)$ has the integral represention

$$
Q(z)=\beta z+\alpha+\int_{\mathbb{R}}\left(\frac{1}{t-z}-\frac{t}{t^{2}+1}\right) d \sigma(t), z \in \mathbb{C} \backslash \mathbb{R},
$$

where $\beta \geq 0, \alpha \in \mathbb{R}$, and $\sigma(t)$ is a nondecreasing function on $\mathbb{R}$ for which

$$
\int_{\mathbb{R}} \frac{d \sigma(t)}{t^{2}+1}<\infty
$$

cf. [5, p. 20], 12]. The fractional linear transformation

$$
L_{\tau}(z)=\frac{z-\tau}{1+\tau z}, \quad \tau \in \mathbb{R} \cup\{\infty\},
$$

leaves the class $\mathbf{N}$ invariant, i.e. for each $Q(z) \in \mathbf{N}$

$$
Q_{\tau}(z)=L_{\tau}(Q(z)) \in \mathbf{N} .
$$

A function $Q(z) \in \mathbf{N}$ belongs to $\mathbf{N}_{1}$ if and only if $Q(z)$ has the integral representation

$$
Q(z)=\gamma+\int_{\mathbb{R}} \frac{d \sigma(t)}{t-z}, z \in \mathbb{C} \backslash \mathbb{R},
$$

where $\gamma \in \mathbb{R}$ and $\sigma(t)$ is a nondecreasing function on $\mathbb{R}$ for which

$$
\int_{\mathbb{R}} \frac{d \sigma(t)}{|t|+1}<\infty
$$

in which case $\gamma=\lim _{y \rightarrow \infty} Q(i y)$; see [12, Theorem S1.3.1]. Note that in this case the integral representation (2.1) reduces to the form (2.4) with

$$
\beta=0, \quad \gamma=\alpha-\int_{\mathbb{R}} \frac{t}{t^{2}+1} d \sigma(t) .
$$

Moreover, $Q(z) \in \mathbf{N}_{0}$ if and only if $Q(z)$ has the integral representation (2.4) and the function $\sigma(t)$ satisfies

$$
\int_{\mathbb{R}} d \sigma(t)<\infty
$$


see [7, Proposition 1.3]. The fractional linear transformation (2.3) leaves the class $\mathbf{N}_{1}$ invariant, with one exception: if $Q(z) \in \mathbf{N}_{1}$ and $\lim _{y \rightarrow \infty} Q(i y)=\gamma$, then $Q_{\tau}(z) \in \mathbf{N}_{1}$ for all $\tau \in \mathbb{R} \cup\{\infty\}$ with $1+\tau \gamma \neq 0$, but for $\tau=-1 / \gamma(\tau=\infty$ when $\gamma=0$ ), it follows that $Q_{\tau}(z) \in \mathbf{N} \backslash \mathbf{N}_{1}$; see [7, Proposition 1.5].

\section{NeW subclasses of Nevanlinna functions}

Let $\widetilde{\mathbf{M}}_{1}$ denote the class of Nevanlinna functions $Q(z) \in \mathbf{N}$ satisfying

$$
\int_{1}^{\infty} \frac{|Q(i y)-c|}{y} d y<\infty
$$

for some $c \in \mathbb{C}$. Due to the symmetry condition $\overline{Q(z)}=Q(\bar{z})$, this is equivalent to the condition

$$
\int_{-\infty}^{-1} \frac{|Q(i y)-\bar{c}|}{|y|} d y<\infty .
$$

The class of all functions $Q(z) \in \mathbf{N}$ for which (3.1) holds with $c \in \mathbb{R}$ will be denoted by $\mathbf{M}_{1}$.

Theorem 3.1. Let $Q(z) \in \widetilde{\mathbf{M}}_{1}$ with the integral representation (2.1). Then:

(i) $\beta=0$;

(ii) the number $c$ in (3.1) is uniquely determined and it satisfies $\operatorname{Im} c \geq 0$;

(iii) if $\lim _{y \rightarrow \infty} Q($ iy $)$ exists, then it is equal to $c$;

(iv) $Q(z) \in \mathbf{M}_{1}$ if and only if (2.5) holds;

(v) if $Q(z) \in \mathbf{M}_{1}$, then $Q(z) \in \mathbf{N}_{1}, \lim _{y \rightarrow \infty} Q($ iy) exists and equals $\gamma$ in (2.4).

Proof. (i) Since $Q(z) \in \mathbf{N}$, it follows from

$$
\beta=\lim _{y \rightarrow \infty} \frac{Q(i y)}{y}=\lim _{y \rightarrow \infty} \frac{Q(i y)-c}{y}
$$

(see [7, (1.7)]) and (3.1) that $\beta=0$.

(ii) Assume that (3.1) holds with $c_{1}$ and $c_{2}$. Then

$$
\left|c_{1}-c_{2}\right| \leq\left|Q(i y)-c_{1}-\left(Q(i y)-c_{2}\right)\right| \leq\left|Q(i y)-c_{1}\right|+\left|Q(i y)-c_{2}\right|
$$

shows that

$$
\int_{1}^{\infty} \frac{\left|c_{1}-c_{2}\right|}{y} d y<\infty
$$

which implies $c_{1}=c_{2}$. Since $Q(z)$ is a Nevanlinna function, it follows from $\operatorname{Im} Q(i y) \geq 0$ for $y>0$ that

$$
-\operatorname{Im} c \leq \operatorname{Im}(Q(i y)-c) \leq|Q(i y)-c|,
$$

and (3.1) implies $\operatorname{Im} c \geq 0$.

The proof of (iii) is similar to the proof of (ii).

(iv) If $Q(z) \in \mathbf{M}_{1}$, then $c \in \mathbb{R}$, and

$$
\operatorname{Im} Q(i y)=\operatorname{Im}(Q(i y)-c) \leq|Q(i y)-c|
$$

implies that $Q(z) \in \mathbf{N}_{1}$, so that (2.5) holds. Conversely, if (2.5) holds, then it follows from (i) and (2.1) that $Q(z)$ has the form (2.4) and that

$$
\gamma=\alpha-\int_{\mathbb{R}} \frac{t}{t^{2}+1} d \sigma(t) \in \mathbb{R}
$$

(see (2.6) ), so that $\lim _{y \rightarrow \infty} Q(i y)=\gamma \in \mathbb{R}$. Hence, by (iii), $c \in \mathbb{R}$ and $Q(z) \in \mathbf{M}_{1}$.

(v) This follows from (iv) and [12, Theorem S1.3.1]. 
As to (iii) in Theorem 3.1, it seems to be unknown if there is a function $Q(z) \in$ $\widetilde{\mathbf{M}}_{1}$ for which $Q(i y)$ does not have a limit as $y \rightarrow \infty$. Observe that $(\mathrm{v})$ in Theorem 3.1 implies that $\mathbf{M}_{1} \subset \mathbf{N}_{1}$. The following example shows that this inclusion is strict.

Example 3.2. Let the function $\sigma(t)$ be defined by

$$
\sigma(t)=\left\{\begin{array}{l}
0 \text { for } t<2 \\
\int_{2}^{t} \frac{d s}{(\log s)^{2}} \text { for } t \geq 2 .
\end{array}\right.
$$

Then

$$
\int_{\mathbb{R}} \frac{d \sigma(t)}{|t|+1} \leq \int_{2}^{\infty} \frac{1}{t(\log t)^{2}} d t<\infty
$$

Hence, the function

$$
Q(z)=\int_{\mathbb{R}} \frac{d \sigma(t)}{t-z}, \quad z \in \mathbb{C} \backslash[2, \infty),
$$

is well defined (i.e. the integral is absolutely convergent), and $Q(z) \in \mathbf{N}_{1}$. Furthermore, $\lim _{y \rightarrow \infty} Q(i y)=0$. Now assume that $Q(z) \in \mathbf{M}_{1}$. Then, by (iii) and (v) of Theorem 3.1, it follows that

$$
\int_{1}^{\infty} \frac{|Q(i y)|}{y} d y<\infty
$$

However, observe that for $y \geq 1$,

$$
\begin{aligned}
\operatorname{Re} Q(i y) & =\int_{2}^{\infty} \frac{t}{\left(t^{2}+y^{2}\right)(\log t)^{2}} d t \\
& \geq 4 \int_{2}^{\infty} \frac{t}{\left(t^{2}+y^{2}\right)\left(\log \left(t^{2}+y^{2}\right)\right)^{2}} d t=\frac{2}{\log \left(y^{2}+4\right)}
\end{aligned}
$$

and thus

$$
\int_{1}^{\infty} \frac{\operatorname{Re} Q(i y)}{y} d y \geq \int_{1}^{\infty} \frac{2}{y \log \left(y^{2}+4\right)} d y \geq \int_{1}^{\infty} \frac{2 y}{y^{2}+4} \frac{1}{\log \left(y^{2}+4\right)} d y=\infty .
$$

Therefore

$$
\int_{1}^{\infty} \frac{|Q(i y)|}{y} d y=\infty
$$

a contradiction. Hence, $Q(z) \notin \mathbf{M}_{1}$. As a consequence, the inclusion $\mathbf{M}_{1} \subset \mathbf{N}_{1}$ is strict.

The introduction of the classes $\widetilde{\mathbf{M}}_{1}$ and $\mathbf{M}_{1}$ is motivated by the fact that, under mild conditions on their coefficients, the Titchmarsh-Weyl functions of various classes of Sturm-Liouville problems (depending rationally on the eigenvalue parameter) in the limit-point case belong to the class $\widetilde{\mathbf{M}}_{1}$ (with $c=0$ ); see [9, proof of Theorem 4.1] and [10, proof of Theorem 5.1].

Let $\widetilde{\mathbf{M}}_{0}$ denote the class of Nevanlinna functions $Q(z) \in \mathbf{N}$ for which

$$
\sup _{y>0} y|Q(i y)-c|<\infty
$$


for some $c \in \mathbb{C}$. It is clear that $\widetilde{\mathbf{M}}_{0} \subset \widetilde{\mathbf{M}}_{1}$ with the same numbers $c$ in (3.1) and (3.2), and that (3.2) is equivalent to

$$
\sup _{y<0}|y||Q(i y)-\bar{c}|<\infty .
$$

Finally, let $\mathbf{M}_{0}$ be the class of functions $Q(z) \in \mathbf{N}$ for which (3.2) holds with $c \in \mathbb{R}$.

Theorem 3.3. Let $Q(z) \in \widetilde{\mathbf{M}}_{0}$ with the integral representation (2.1). Then:

(i) $\beta=0$;

(ii) the number $c$ in (3.2) is uniquely determined, and it satisfies $\operatorname{Im} c \geq 0$;

(iii) $\lim _{y \rightarrow \infty} Q($ iy) exists, and it is equal to $c$;

(iv) $Q(z) \in \mathbf{N}_{0}$ if and only if (2.7) holds;

(v) if $Q(z) \in \mathbf{M}_{0}$, then $Q(z) \in \mathbf{N}_{0}$, $\lim _{y \rightarrow \infty} Q($ iy) exists and equals $\gamma$ in (2.4).

Proof. (i) and (ii) follow from Theorem 3.1 since $\widetilde{\mathbf{M}}_{0} \subset \widetilde{\mathbf{M}}_{1}$. that

(iii) The statement follows immediately from the definition (3.2), which implies

$$
\lim _{y \rightarrow \infty}|Q(i y)-c|=0 .
$$

(iv) and (v) can be shown as in the proof of Theorem 3.1 ,

Corollary 3.4. The following identity holds:

$$
\mathbf{M}_{0}=\mathbf{N}_{0} \text {. }
$$

Proof. The inclusion $\mathbf{M}_{0} \subset \mathbf{N}_{0}$ is clear from the definitions. Now let $Q(z) \in \mathbf{N}_{0}$. Then (2.4) and (2.7) imply that for all $y>0$

$$
\begin{aligned}
y|Q(i y)-\gamma| & =\left|\int_{\mathbb{R}} \frac{y}{t-i y} d \sigma(t)\right| \\
& \leq \int_{\mathbb{R}} \frac{y}{\sqrt{t^{2}+y^{2}}} d \sigma(t) \leq \int_{\mathbb{R}} d \sigma(t)<\infty .
\end{aligned}
$$

Hence $Q(z) \in \mathbf{M}_{0}$, proving the inclusion $\mathbf{N}_{0} \subset \mathbf{M}_{0}$.

Example 3.5. For $c \in \mathbb{C}, \operatorname{Im} c>0$, define the function $Q(z)$ by

$$
Q(z)= \begin{cases}c & \text { if } \operatorname{Im} z>0, \\ \bar{c} & \text { if } \operatorname{Im} z<0 .\end{cases}
$$

Then clearly $Q(z) \in \mathbf{N}$ and, in fact, $Q(z) \in \widetilde{\mathbf{M}}_{0}$. However, $Q(z) \notin \mathbf{N}_{1}$.

If $Q_{0}(z)$ is a Nevanlinna function and $c \in \mathbb{C}$ with $\operatorname{Im} c \geq 0$, then also

$$
Q(z)= \begin{cases}c+Q_{0}(z) & \text { if } \operatorname{Im} z>0 \\ \bar{c}+Q_{0}(z) & \text { if } \operatorname{Im} z<0\end{cases}
$$

is a Nevanlinna function. Clearly, if $c \in \mathbb{R}$, then $Q(z) \in \mathbf{N}$ implies $Q_{0}(z)=$ $Q(z)-c \in \mathbf{N}$. However, the situation is different for $c \notin \mathbb{R}$, as the following example shows.

Example 3.6. Let $\alpha=\beta=0$ and $d \sigma(t)=(1-\cos t) d t$ in (2.1). Then the integrability condition (2.2) is satisfied and the Nevanlinna function $Q(z)$ in (2.1) is given by

$$
Q(z)=\int_{\mathbb{R}}\left(\frac{1}{t-z}-\frac{t}{t^{2}+1}\right)(1-\cos t) d t, \quad z=x+i y, \quad y>0 .
$$


In order to calculate this absolutely convergent integral, observe that

$$
\begin{aligned}
Q(z)=\int_{\mathbb{R}} & \left(\frac{t-x}{(t-x)^{2}+y^{2}}-\frac{t}{t^{2}+1}\right)(1-\cos t) d t \\
& +i y \int_{\mathbb{R}} \frac{1}{(t-x)^{2}+y^{2}}(1-\cos t) d t, \quad z=x+i y, \quad y>0 .
\end{aligned}
$$

In (3.3) replace $\cos t$ by $e^{i t}$ and apply the calculus of residues. One obtains

$$
Q(z)=\pi e^{-y} \sin x+\pi i\left(1-e^{-y} \cos x\right), \quad z=x+i y, \quad y>0,
$$

and, in particular,

$$
Q(i y)=\pi i\left(1-e^{-y}\right), \quad y>0
$$

Hence $Q(i y)-\pi i=-\pi i e^{-y}$, which shows that $Q(z) \in \widetilde{\mathbf{M}}_{0}$ with $c=\pi i$, and $\lim _{y \rightarrow \infty} Q(i y)=c \notin \mathbb{R}$. Furthermore this shows that $Q(z)-c \notin \mathbf{N}$.

\section{Characterizations of $\mathbf{M}_{1}$}

Recall that if $Q(z) \in \mathbf{M}_{1}$, i.e. (3.1) holds with $c \in \mathbb{R}$, then $Q(z)$ has the integral representation (2.4), where $\sigma(t)$ is a nondecreasing function which satisfies (2.5) and where $\gamma=c$. It has been shown that the class $\mathbf{M}_{1}$ is a proper subclass of $\mathbf{N}_{1}$. A precise characterization of $\mathbf{M}_{1}$ is now given.

Proposition 4.1. $Q(z)$ belongs to $\mathbf{M}_{1}$ if and only if $Q(z)$ belongs to $\mathbf{N}_{1}$, and the measure $d \sigma$ in the integral representation (2.4) satisfies

$$
\int_{1}^{\infty} \frac{1}{y}\left|\int_{\mathbb{R}} \frac{t}{t^{2}+y^{2}} d \sigma(t)\right| d y<\infty
$$

Proof. The statement is an immediate consequence of (1.1) and

$$
|Q(i y)-\gamma| \leq\left|\int_{\mathbb{R}} \frac{t}{t^{2}+y^{2}} d \sigma(t)\right|+\operatorname{Im} Q(i y) \leq 2|Q(i y)-\gamma|
$$

since $\mathbf{M}_{1} \subset \mathbf{N}_{1}$ by Theorem 3.1

Theorem 4.2. Let $Q(z) \in \mathbf{M}_{1}$ have the integral representation (2.4). Then

$$
\lim _{r \rightarrow \infty} \int_{\mathbb{R}} \frac{1}{t} \log \frac{t^{2}+1}{\left(\frac{t}{r}\right)^{2}+1} d \sigma(t)
$$

exists and is finite.

Proof. Proposition 4.1 implies that

$$
\lim _{r \rightarrow \infty} \int_{1}^{r} \frac{1}{y}\left(\int_{\mathbb{R}} \frac{t}{t^{2}+y^{2}} d \sigma(t)\right) d y
$$

exists and is finite. Since

$$
\int_{1}^{r} \frac{1}{y} \int_{\mathbb{R}} \frac{|t|}{t^{2}+y^{2}} d \sigma(t) d y<\infty
$$

the above integral can be rewritten by Fubini's theorem as

$$
\int_{1}^{r} \frac{1}{y}\left(\int_{\mathbb{R}} \frac{t}{t^{2}+y^{2}} d \sigma(t)\right) d y=\int_{\mathbb{R}} t\left(\int_{1}^{r} \frac{1}{y\left(t^{2}+y^{2}\right)} d y\right) d \sigma(t) .
$$


Then

$$
\int_{1}^{r} \frac{1}{y\left(t^{2}+y^{2}\right)} d y=\frac{1}{t^{2}} \int_{1}^{r}\left(\frac{1}{y}-\frac{y}{t^{2}+y^{2}}\right) d y=\frac{1}{2 t^{2}} \log \frac{t^{2}+1}{\left(\frac{t}{r}\right)^{2}+1}
$$

proves the statement about the limit in (4.1).

Observe that if $\sigma(t)$ is a nondecreasing function on $\mathbb{R}$ such that

$$
\int_{\mathbb{R}} \frac{\log \left(t^{2}+1\right)}{|t|} d \sigma(t)<\infty,
$$

then the integrability condition (2.5) is satisfied.

Theorem 4.3. Let $Q(z) \in \mathbf{N}$ have the integral representation (2.4) with $\gamma \in \mathbb{R}$, and assume that the integrability condition (4.2) holds. Then $Q(z) \in \mathbf{M}_{1}$.

Proof. An obvious modification of the argument in the proof of Theorem 4.2 shows that

$$
\int_{1}^{\infty} \frac{1}{y}\left(\int_{\mathbb{R}}\left|\frac{t}{t^{2}+y^{2}}\right| d \sigma(t)\right) d y<\infty
$$

is equivalent to (4.2). An application of Proposition 4.1] gives that $Q(z) \in \mathbf{M}_{1}$.

The conditions (4.1) and (4.2) are clearly integrability conditions at $\pm \infty$. Therefore, if $d \sigma$ is supported on a half-line $(-\infty, b]$ or $[a, \infty)$, one only has to verify the conditions (4.1) and (4.2) when the integration takes place over $[0, \infty)$ or $(-\infty, 0]$, respectively; in this case the conditions (4.1) and (4.2) are equivalent.

Corollary 4.4. Let $Q(z) \in \mathbf{N}$ and assume that the domain of holomorphy of $Q(z)$ contains $\mathbb{C} \backslash(-\infty, a]$ or $\mathbb{C} \backslash[a, \infty)$ for some $a \in \mathbb{R}$. Then $Q(z) \in \mathbf{M}_{1}$ if and only if $Q(z)$ has the integral representation (2.4), where $\gamma \in \mathbb{R}$ and $\sigma$ satisfies (4.2).

If there is some $y_{0}>0$ such that $\operatorname{Re} Q(i y)-\gamma$ does not change sign for $y>y_{0}$, all steps in the proof of Theorem 4.2 can be reversed.

Corollary 4.5. Let $Q(z) \in \mathbf{N}_{1}$ and let $y_{0}>0$ be such that $\operatorname{Re} Q(i y)-\gamma$ does not change sign for $y>y_{0}$. Then $Q(z) \in \mathbf{M}_{1}$ if and only if

$$
\lim _{r \rightarrow \infty} \int_{\mathbb{R}} \frac{1}{t} \log \frac{t^{2}+1}{\left(\frac{t}{r}\right)^{2}+1} d \sigma(t)
$$

exists and is finite.

\section{Fractional linear transformations}

Proposition 5.1. Assume that $Q(z) \in \mathbf{M}_{1}$, i.e., assume that $Q(z) \in \mathbf{N}$ satisfies (3.1) with $c \in \mathbb{R}$ and $\lim _{y \rightarrow \infty} Q(i y)=c$. Then for all $\tau \in \mathbb{R} \cup\{\infty\}$ with $1+\tau c \neq 0$, one has

$$
Q_{\tau}(z) \in \mathbf{M}_{1} \quad \text { and } \quad \lim _{y \rightarrow \infty} Q_{\tau}(i y)=L_{\tau}(c) .
$$

Moreover, for the exceptional value of $\tau=-1 / c(\tau=\infty$ when $c=0)$,

$$
Q_{\tau}(z) \notin \mathbf{M}_{1} \text {. }
$$


Proof. Consider for $\tau \in \mathbb{R}$ and $1+\tau c \neq 0$ the identity

$$
Q_{\tau}(i y)-L_{\tau}(c)=(Q(i y)-c) \frac{1+\tau^{2}}{(1+\tau c)(1+\tau Q(i y))}
$$

and observe that

$$
\frac{1+\tau^{2}}{(1+\tau c)(1+\tau Q(i y))}=\frac{1+\tau^{2}}{(1+\tau c)^{2}}+o(1), \quad y \rightarrow \infty .
$$

It follows from (5.1) and (5.2) that

$$
\int_{\mathbb{R}} \frac{1}{y}\left|Q_{\tau}(i y)-L_{\tau}(c)\right| d y<\infty
$$

so that $Q_{\tau}(z) \in \mathbf{M}_{1}$. When properly interpreted, this result extends to the case $\tau=\infty$ and $c \neq 0$. Finally, for the exceptional value of $\tau$ it follows that

$$
\lim _{y \rightarrow \infty} Q_{\tau}(i y)=L_{\tau}(c)=\infty .
$$

In this case $Q_{\tau}(z)$ cannot have the representation (2.4), which implies that $Q_{\tau}(z) \notin$ $\mathbf{N}_{1}$ and hence $Q_{\tau}(z) \notin \mathbf{M}_{1}$.

Remark 5.2. If $Q(z) \in \widetilde{\mathbf{M}}_{1}$ with $c \in \mathbb{C} \backslash \mathbb{R}$ and $\lim _{y \rightarrow \infty} Q(i y)=c$, then $Q_{\tau}(z) \in \widetilde{\mathbf{M}}_{1}$ for all $\tau \in \mathbb{R} \cup\{\infty\}$. A similar statement is valid for functions in the class $\widetilde{\mathbf{M}}_{0}$.

In Sturm-Liouville problems the functions $Q_{\tau}(z)$ appear as Titchmarsh-Weyl coefficients associated with appropriate boundary conditions. The exceptional value $\tau=-1 / \gamma$ in Proposition 5.1 corresponds to the generalized Friedrichs extension; this extension coincides with the usual Friedrichs extension if the underlying symmetric operator is semibounded; cf. [6], [7, 8], [9], [10].

\section{REFERENCES}

[1] L. de Branges, "Some Hilbert spaces of entire functions", Trans. Amer. Math. Soc., 96 (1960), 259-295. MR0133455(24:A3289a)

[2] L. de Branges, "Some Hilbert spaces of entire functions II", Trans. Amer. Math. Soc., 99 (1961), 118-152. MR0133456 (24:A3289b)

[3] L. de Branges, "Some Hilbert spaces of entire functions III", Trans. Amer. Math. Soc., 100 (1961), 73-115. MR0133457(24:A3289c)

[4] L. de Branges, "Some Hilbert spaces of entire functions IV", Trans. Amer. Math. Soc., 105 (1962), 43-83. MR0143016 (26:583)

[5] W.F. Donoghue, Monotone matrix functions and analytic continuation, Springer-Verlag, Berlin-Heidelberg-New York, 1974. MR0486556 (58:6279)

[6] S. Hassi, M. Kaltenbäck, and H.S.V. de Snoo, "Triplets of Hilbert spaces and Friedrichs extensions associated with the subclass $\mathbf{N}_{1}$ of Nevanlinna functions", J. Operator Theory, 37 (1997), 155-181. MR.1438205 (98c:47013)

[7] S. Hassi, H. Langer, and H.S.V. de Snoo, "Selfadjoint extensions for a class of symmetric operators with defect numbers $(1,1) ", 15$ th OT Conference Proc., (1995), 115-145. MR1421120 (97m:47024)

[8] S. Hassi, M. Möller, and H.S.V. de Snoo, "Differential operators and spectral functions", Proceedings of UMC-2001, 11, 313-322 (2002).

[9] S. Hassi, M. Möller, and H.S.V. de Snoo, "Sturm-Liouville operators and their spectral functions", J. Math. Anal. Appl., 282 (2003), 584-602. MR1989113(2004e:47073)

[10] S. Hassi, M. Möller, and H.S.V. de Snoo, "Singular Sturm-Liouville problems whose coefficients depend rationally on the eigenvalue parameter", J. Math. Anal. Appl., 295 (2004), 258-275. MR2064424 (2005c:34046)

[11] I.S. Kac, "On integral representations of analytic functions mapping the upper halfplane into itself", Uspekhi Mat. Nauk, 11 (1956), 139-144 (Russian). MR0080745 (18:293b) 
[12] I.S. Kac and M.G. Krel̆n, " $R$-functions - analytic functions mapping the upper halfplane into itself", Supplement I to the Russian edition of F.V. Atkinson, Discrete and continuous boundary problems, Mir, Moscow, 1968 (Russian) (English translation: Amer. Math. Soc. Transl. Ser. 2, 103 (1974), 1-18). MR0328627 (48:6969)

[13] H. Winkler, "The inverse spectral problem for canonical systems", Integral Equations Operator Theory, 22 (1995), 360-374. MR1337383 (96f:34020)

Department of Mathematics and Statistics, University of Vaasa, P.O. Box 700, 65101 VAASA, FinLAND

E-mail address: sha@uwasa.fi

Department of Mathematics, University of the Witwatersrand, Wits, 2050, South AFRICA

E-mail address: manfred@maths.wits.ac.za

Department of Mathematics and Computing Science, University of Groningen, P.O. Box 800, 9700 AV Groningen, Nederland

E-mail address: desnoo@math.rug.nl 